\title{
A rapid method to quantify casein in fluid milk by front-face fluorescence spectroscopy combined with chemometrics
}

\author{
Yizhou B. Ma and Jayendra. K. Amamcharla* (1) \\ Department of Animal Sciences and Industry/Food Science Institute, Kansas State University, Manhattan 66506
}

\section{ABSTRACT}

Casein in fluid milk determines cheese yield and affects cheese quality. Traditional methods of measuring casein in milk involve lengthy sample preparations with labor-intensive nitrogen-based protein quantifications. The objective of this study was to quantify casein in fluid milk with different casein-to-crude-protein ratios using front-face fluorescence spectroscopy (FFFS) and chemometrics. We constructed calibration samples by mixing microfiltration and ultrafiltration retentate and permeate in different ratios to obtain different casein concentrations and casein-to-crude-protein ratios. We developed partial least squares regression and elastic net regression models for casein prediction in fluid milk using FFFS tryptophan emission spectra and reference casein contents. We used a set of 20 validation samples (including raw, skim, and ultrafiltered milk) to optimize and validate model performance. We externally tested another independent set of 20 test samples (including raw, skim, and ultrafiltered milk) by root mean square error of prediction (RMSEP), residual prediction deviation (RPD), and relative prediction error (RPE). The RMSEP for casein content quantification in raw, skim, and ultrafiltered milk ranged from 0.12 to $0.13 \%$, and the RPD ranged from 3.2 to 3.4. The externally validated error of prediction was comparable to the existing rapid method and showed practical model performance for quality-control purposes. This FFFS-based method can be implemented as a routine quality-control tool in the dairy industry, providing rapid quantification of casein content in fluid milk intended for cheese manufacturing.

Key words: casein, rapid method, partial least square regression, elastic net regression

Received April 27, 2020.

Accepted August 24, 2020.

*Corresponding author: jayendra@ksu.edu

\section{INTRODUCTION}

Improving cheese yield is a constant pursuit among cheese manufacturers. Dairy food scientists have identified multiple factors that can influence cheese yield. Milk compositions - namely the amount of casein and fat-have been highlighted in multiple studies as indicators that determine cheese yield (Barbano and Sherbon, 1984; Emmons and Modler, 2010). Several determinants of cheese yield include curd firmness, syneresis rate, and moisture retention, and these qualities of the cheese curd have been partially linked to the casein content of the cheese milk (Cipolat-Gotet et al., 2013). Therefore, standardization of cheese milk has become a common practice in cheese manufacturing. By adjusting the casein-to-fat ratio, standardized milk can maximize cheese yield without losing excessive fat and casein into whey (Lucey and Kelly, 1994). Moreover, with advancements in membrane processing technologies, cheese can be produced from UF and microfiltered (MF) milk. Both UF and MF milk contain more casein than regular cheese milk, which can increase cheese yield and improve vat utilization (Kumar et al., 2013).

The current standard method of casein measurement involves isoelectrically precipitating casein at $\mathrm{pH} 4.6$ in milk and separating the casein from the non-casein fraction by filtration according to AOAC International (2016) standard methods (990.20 and 990.21). Casein content can be directly measured from isolated casein solids or indirectly calculated as the difference between total protein and non-casein proteins using a Kjeldahlbased method. The Kjeldahl-based method has good repeatability and reproducibility and has served as the industry standard method for casein quantification since 1938 (Rowland, 1938; Lynch et al., 1998). However, this quantification process is laborious and time-consuming, and it uses multiple hazardous chemical reagents. Dairy food researchers have proposed 2 general alternative approaches to measuring casein based on separation techniques and infrared spectroscopies. High-performance liquid chromatography methods based on reverse phase, gel permeation, and size 
exclusion have been developed to quantify caseins in skim and raw milk (Dimenna and Segall, 1981; van der Ven et al., 2001; Bonfatti et al., 2008). Similarly, a capillary electrophoresis-based method has been developed to quantify whey protein and casein in milk (Recio and Olieman, 1996). Although the primary goal of these methods was protein separation, quantification of casein can also be achieved using appropriate standards. Infrared spectroscopic methods of casein quantification have been developed with the help of multivariate statistical models. One early attempt at near-infrared measurement of casein was based on an indirect approach, taking the difference between total protein and serum phase protein (Barbano and Dellavalle, 1987). With the advancement of Fourier-transform infrared spectroscopy (FTIR), Hewavitharana and van Brakel (1997) and Luginbühl (2002) have both developed and validated casein quantification with FTIR and multivariate statistical models.

The milk samples used in previous spectroscopic studies had similar casein-to-crude-protein ratios (CN:CP), making prediction dependent on the collinearity between casein and CP (Baum et al., 2016). With consistent $\mathrm{CN}$ : $\mathrm{CP}$, casein measurement becomes a secondary quantification by measuring CP. Foss Electric (Hillerød, Denmark) has also implemented calibration options for measuring casein in fluid milk using its FTIR models MilkoScan FT 120 and MilkoScan FT2 (Foss Electric, 1997; Baum et al., 2016). However, published FTIR-based methods have reported only crossvalidation results. The lack of external validation has made it difficult to fully examine the robustness and practicality of this method when measuring unknown samples.

Multiple intrinsic fluorophores in milk are suitable for fluorescence spectroscopic analysis. Front-face fluorescence spectroscopy (FFFS) is known for its sensitivity and ability to analyze turbid samples. Tryptophan, a commonly studied fluorophore in milk, can be used to measure milk coagulation, degrees of heat treatment, and dairy powder solubility during storage (Herbert et al., 1999; Kulmyrzaev et al., 2005; Babu and Amamcharla, 2018). Herbert et al. (1999) studied tryptophan emission spectra and characterized the acid coagulation process in milk. The study indicated that in acidic conditions, casein in milk yielded fluorescence spectral differences from milk with a native $\mathrm{pH}$ as a result of structural changes in casein, such as solubilization of micellar calcium phosphate and partial micellar disintegration. These structural changes in casein affect surface tryptophan exposure and lead to an increase in tryptophan fluorescence intensity. Ma et al. (2019) measured acid-precipitated casein tryptophan fluores- cence in raw milk and established multivariate calibration models to quantify casein. This study extends the previous study to develop and externally validate a FFFS-based method to quantify casein in raw, skim, and $\mathrm{UF}$ milk with various $\mathrm{CN}$ :CP.

\section{MATERIALS AND METHODS}

\section{Experimental Approach}

We constructed calibration samples by mixing permeates and retentates obtained from UF and MF pasteurized skim milk in different ratios to obtain a range of different casein concentrations and CN:CP. We developed multivariate calibration models using tryptophan emission spectra and reference values of casein content and CN:CP based on the Kjeldahl method. We subsequently optimized the calibration models using a set of validation samples including raw, skim, and UF milk and evaluated the final model performance for casein and CN:CP measurement using another independent set of test samples including raw, skim, and UF milk. Detailed methods are described in the following sections.

\section{Calibration, Validation, and Test Samples}

The UF retentate and permeate (about $5 \times$ concentrated) made from 1 lot of pasteurized skim milk were donated by a commercial milk protein concentrate manufacturer in the United States. The MF retentate and permeate (about $3 \times$ concentrated) from 1 lot of pasteurized skim milk were donated by the Southeast Dairy Foods Research Center (Raleigh, NC). Detailed UF and MF sample preparation is described in Carter et al. (2018). Both UF and MF milk fractions arrived under refrigerated conditions and were analyzed for casein content based on the Kjeldahl method (AOAC International, 2016; methods 990.20 and 990.21).

After the casein content was measured in the UF and MF permeate and retentate, the UF and MF retentates were diluted with varying amounts of UF and MF permeates to vary casein content and $\mathrm{CN}$ : $\mathrm{CP}$ in the calibration samples. We prepared 30 calibration samples $\left(\mathrm{n}_{\text {cal }}=30\right)$, with casein content ranging from 1.21 to $4.45 \%$ and $\mathrm{CN}$ :CP ranging from 0.66 to 0.88 , and we used them to develop the calibration model (Table 1).

For validation and test samples, we purchased 10 samples of pasteurized skim milk and 10 samples of UF milk with different production days from local supermarkets. An additional 20 raw milk samples from individual cows were randomly collected from the Kansas State University Dairy Cattle Teaching and Research 
Table 1. Mean (range) protein fractions of calibration samples $(\mathrm{n}=30)$, ultrafiltered milk $(\mathrm{n}=10)$, pasteurized skim milk $(\mathrm{n}=10)$, and raw milk $(\mathrm{n}=20)$

\begin{tabular}{lcccr}
\hline & & & \multicolumn{2}{c}{ Validation and test samples } \\
\cline { 3 - 5 } Item & Calibration & & & \\
& samples & Raw milk & Pasteurized skim milk & \multirow{2}{*}{ Ultrafiltered milk } \\
\hline CP (\%) & $3.29(1.82-5.17)$ & $2.91(2.57-3.34)$ & $3.16(2.75-3.42)$ & $3.77(3.54-3.93)$ \\
Casein (\%) & $2.57(1.21-4.45)$ & $2.25(1.88-2.66)$ & $2.37(2.03-2.64)$ & $3.17(2.90-3.34)$ \\
NPN (\% protein-equivalent) & $0.14(0.10-0.26)$ & $0.12(0.09-0.14)$ & $0.12(0.10-0.14)$ & $0.17(0.14-0.20)$ \\
CN:CP ratio & $0.77(0.66-0.88)$ & $0.77(0.73-0.80)$ & $0.76(0.70-0.79)$ & $0.84(0.82-0.86)$ \\
\hline
\end{tabular}

Unit (Manhattan, KS). The validation and test samples $\left(\mathrm{n}_{\mathrm{val} / \text { test }}=40\right)$ were stored at $5^{\circ} \mathrm{C}$ until further analysis.

\section{Reference Measurement of Casein and Calibration Sample Preparation}

We analyzed CP and NPN in the UF and MF retentate, permeate, and validation and test milk samples using AOAC International (2016) standard methods (990.20 and 990.21). Because of the high protein content in the MF and UF retentate, we measured noncasein nitrogen according to the method of Zhang and Metzger (2011). We determined casein content from the difference between $\mathrm{CP}$ and noncasein nitrogen content, multiplied by 6.38 . We calculated $\mathrm{CN}$ :CP using casein content divided by $\mathrm{CP}$ to represent the proportion of casein in relationship to the total protein of the milk sample.

\section{Tryptophan Fluorescence Collection for Calibration and Validation Samples}

Based on preliminary studies, completely precipitating casein at $\mathrm{pH} 4.6$ yielded distinctive spectra compared with the rest of the $\mathrm{pH}$-adjusted and native samples. The FFFS spectral collection was achieved according to Ma et al. (2019). Prior to FFFS measurement, $7 \mathrm{~mL}$ of sample was taken in a 10-mL test tube and mixed with $0.6 \mathrm{~mL}$ of $10 \%$ acetic acid (Fisher Scientific, Hampton, $\mathrm{NH})$ to ensure a $\mathrm{pH}$ of $4.60 \pm 0.05$. The mixture was vortexed for $15 \mathrm{~s}$ and transferred immediately into a quartz cuvette (Starna Cells Inc., Atascadero, CA), ensuring no phase separation. Tryptophan emission spectra were immediately acquired using a spectrofluorimeter fitted with a $1 \%$ attenuator (LS-55; Perkin Elmer, Waltham, MA) at an excitation wavelength of 280 $\mathrm{nm}$ and an emission scan of 300 to $440 \mathrm{~nm}$, with a scan speed of $300 \mathrm{~nm} / \mathrm{min}$. We performed triplicate measurements on freshly precipitated calibration samples at $25^{\circ} \mathrm{C}$ and averaged them to improve signal-to-noise ratio. In total, we collected tryptophan fluorescence spectra on 30 calibration and 40 validation and test samples to develop the chemometric model.

\section{Chemometric Model Development, Optimization, and Validation}

Developing chemometric models involves optimization and testing of the finalized models (Bevilacqua et al., 2017). The detailed model-development approach followed in this study can be found in Figure 1. We developed calibration models using FFFS tryptophan spectra, reference casein content, and the $\mathrm{CN}: \mathrm{CP}$ of the 30 calibration samples. The 40 validation and test samples were randomly partitioned into a validation set $\left(\mathrm{n}_{\text {val }}=20\right)$ and a test set $\left(\mathrm{n}_{\text {test }}=20\right)$. A summary of the casein content and $\mathrm{CN}: \mathrm{CP}$ of the validation and test sets can be found in Table 1. We used the validation set to validate and optimize the preliminary models, and we used the test set to evaluate the optimized model for quantification of casein content and $\mathrm{CN}$ :CP. The quantification results from the test set provided estimates of the future performance of the developed model.

Spectral Preprocessing and Construction of Calibration Models. Preprocessing tools such as normalization, derivation, and smoothing are commonly used to reduce drift noise and reveal spectral overlays before model development (Brown et al., 2000). In this study, raw fluorescence spectra were transformed using Savitzky-Golay smoothing and first derivative algorithms with 9-point neighbor values to reduce the spectral noise from directly measuring turbid milk samples and reveal additional spectral information (Savitzky and Golay, 1964).

We developed supervised prediction models using partial least squares regression (PLSR) and elastic net regression (ENR). In chemometrics, PLSR is a popular method for relating 2 data matrices using a linear multivariate model, capable of handling large number variables with noise and collinearity (Wold et al., 2001). Elastic net regression is a type of panelized linear regression with the ability to eliminate and shrink variable contributions in multivariate models (Chen et al., 2014). Chemometric researchers have applied both PLSR and ENR to model spectral data because of their ability to handle large numbers of predictors (Filzmoser et al., 2012). The model input consisted of the smoothed 
or first derivative of the tryptophan emission spectra, and casein content was predicted independently using the preprocessed spectra. In this study, we considered up to 15 latent variables in the initial model development of PLSR and used them as model optimization parameters. For ENR, the elastic net parameter $(\alpha)$ and regularization parameter $(\lambda)$ were considered optimization parameters. In this study, we used an increment of 0.1 for $\alpha$ optimization, using leave-oneout cross-validation to find the best-performing $\lambda$. A total of 10 models from ENR were produced from the calibration step, and they were later optimized by the validation set for the optimal $\alpha$ value.

Model Optimizations. We optimized the established calibration models by predicting the validation set. We evaluated model performance using root mean square error of validation (RMSEV) and the coeffi-

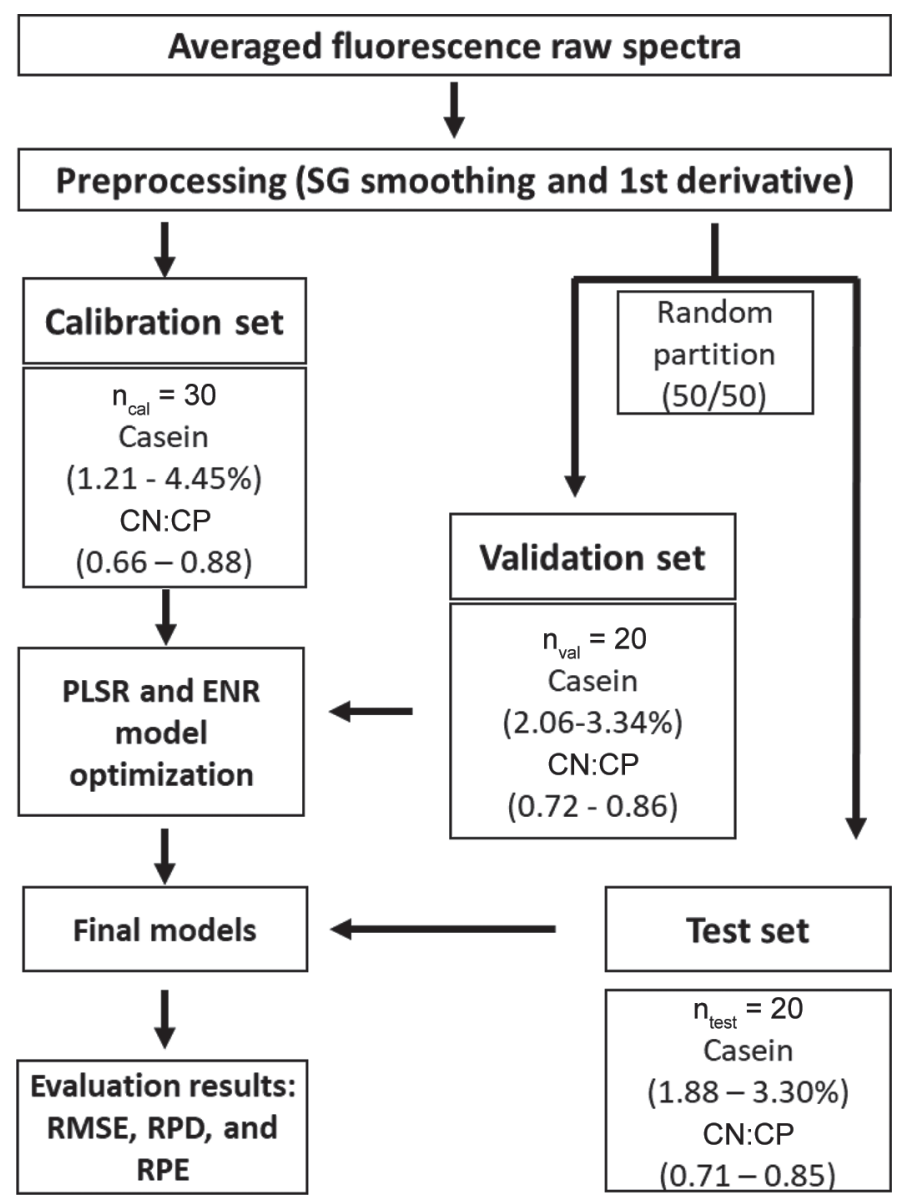

Figure 1. Chemometric model development overview with casein (range) and $\mathrm{CN}: \mathrm{CP}$ (range). $\mathrm{CN}: \mathrm{CP}=$ casein-to-crude-protein ratio; $\mathrm{ENR}=$ elastic net regression; $\mathrm{n}_{\mathrm{cal}}=$ number of calibration samples; $\mathrm{n}_{\mathrm{val}}=$ number of validation samples; $\mathrm{n}_{\text {test }}=$ number of test samples; PLSR $=$ partial least squares regression; $\mathrm{RMSE}=$ root mean square error; $\mathrm{RPD}=$ residual prediction deviation $\mathrm{RPE}=$ relative prediction error; $\mathrm{SG}=$ Savitzky-Golay. cient of determination $\left(\mathrm{R}^{2}\right)$ between the reference and predicted values. We selected optimization parameters for PLSR (number of latent variables) and ENR ( $\alpha$ and $\lambda$ values) based on the lowest RMSEV. The $\mathrm{R}^{2}$ evaluated the linearity of the model prediction to the reference values, and we used calibration transfer based on linear models to correct the estimated bias from the preliminary PLSR or ENR predictions. We recorded the optimal parameters of PLSR and ENR and used them for the final test set predictions.

Model Performance Evaluation. Model evaluation was achieved by predicting the test set using the finalized PLSR and ENR models. We evaluated the final model performance using root mean square error of prediction (RMSEP), showing the difference between predicted values and reference values. We calculated residual prediction deviation (RPD) from the RMSEP divided by the standard deviation of the reference values and used it as a parameter to estimate the model's prediction power. We calculated relative prediction errors (RPE) by dividing the average reference values by the RMSEP to evaluate the relative error of the prediction to the reference method. We conducted spectral preprocessing, statistical model building, and evaluation using an in-house program developed in the $\mathrm{R}$ programming language with the Caret, pls, and glmnet packages (Mevik and Wehrens, 2007; Kuhn, 2008; Friedman et al., 2010; R Core Team, 2016).

\section{RESULTS AND DISCUSSION}

\section{Reference Casein and CN:CP Measurements}

In general, the average casein content in milk is 2.6 to $2.8 \%$ (Walstra and Jenness, 1984; Fox et al., 1998). However, average casein content can be affected by season, diet, and genetic variations in dairy cows (Lin et al., 2017). Lin et al. (2017) evaluated the casein content of pooled pasteurized skim milk from Holstein Friesians over 1 year and found that casein content ranged from 2.61 to $3.02 \%$. Chen et al. (2014) monitored pooled raw milk for 1 year and found that casein content ranged from 2.08 to $2.52 \%$. With the recent popularity of highprotein beverages, UF milk as a consumer product has entered the market. According to a high-protein milk application developed by Ur-Rehman et al. (2017), casein in UF and delactosed milk can range from 2 to $8 \%$ during production, and for the finished product, CP content can range from 4.9 to $5.2 \%$. Although casein content is not specified for the finished product, it is assumed to be less than the reported total protein content. Table 1 summarizes the protein fractions of UF milk obtained for this study. For the 10 commercial UF milk samples we obtained, casein content ranged from 
2.90 to $3.34 \%$, and CP ranged from 3.54 to $3.93 \%$. For the commercial pasteurized skim milk samples, casein content ranged from 2.03 to $2.64 \%$, and $\mathrm{CP}$ ranged from 2.75 to $3.42 \%$. Casein content for the raw milk samples in this study ranged from 1.88 to $2.66 \%$, and $\mathrm{CP}$ ranged from 2.57 to $3.39 \%$. The casein content variation observed in this study suggested that the calibration range for measuring casein needed to cover the casein range for raw, skim, and UF milk. In Table 1, the casein content of the calibration samples ranged from 1.21 to $4.45 \%$, providing a sufficient calibration range for measuring casein in these milk types.

The casein content variation in milk can also cause variations in CN:CP. Lin et al. (2017) reported that the CN:CP for pasteurized skim milk from Holstein Friesian cows ranged from 0.75 to 0.81 over 1 yr of observation. According to Schaar et al. (1985), genetic variants affected $\kappa-\mathrm{CN}$ and $\beta$-LG synthesis during lactation, which led to variations in casein numbers $(\mathrm{CN}: \mathrm{CP} \times 100)$ and cheese composition (Lundén et al., 1997). We also observed variations in $\mathrm{CN}: \mathrm{CP}$ in this study. The $\mathrm{CN}$ :CP variation ranged from 0.82 to 0.86 in UF milk, 0.71 to 0.79 in pasteurized skim milk, and 0.73 to 0.80 in raw milk (Table 1 ). The variation in milk samples required a set of calibration samples that covered the target $\mathrm{CN}$ : $\mathrm{CP}$ range. Table 1 showed that the calibration sample range was obtained by mixing various amount of UF and MF retentate and permeate, which produced $\mathrm{CN}$ : $\mathrm{CP}$ of 0.66 to 0.88 . With the wide range of $\mathrm{CN}: \mathrm{CP}$ in the calibration set, we were able to avoid the collinearity effects between casein and CP and ensure robust casein predictions in various types of milk.

\section{Tryptophan Fluorescence Emission Spectra of Calibration Samples}

We collected tryptophan fluorescence emission spectra of acid-precipitated calibration samples using FFFS. The emission maxima $(\boldsymbol{\lambda} \max )$ of the calibration samples $\left(\mathrm{n}_{\text {cal }}=30\right)$ ranged from 338 to $341 \mathrm{~nm}$. According to Andersen and Mortensen (2008), emission maxima of approximately $340 \mathrm{~nm}$ confirm the fluorophore to be tryptophan. The calibration set varied in terms of casein content and CN:CP (Table 1) and led to differences in tryptophan emission intensity. Figure 2A shows 4 representative calibration samples with low CP (samples I and II) and high CP (samples III and IV). In the samples with low CP, the casein content in sample I (1.6\%) was less than in sample II $(2.1 \%)$. The non-casein nitrogen levels for samples I and II were also different, at 0.9 and $0.4 \%$, respectively. The CP levels for samples I and II were very similar, at $2.5 \%$. In Figure 2B, the tryptophan emission spectra of samples
I and II appeared to have large fluorescence emission intensity differences, with a $\lambda \max$ increase of $23.3 \%$. On the other hand, a similar amount of casein was present in samples II and III (2.1\%), but their CP contents were different, because the non-casein nitrogen of sample III was higher than that of sample II (1.3 and $0.4 \%$, respectively). The emission spectra of samples II and III appeared to be similar, with a $2.3 \%$ change in $\lambda$ max. Samples III and IV had a similar amount of CP $(3.4 \%)$, but different casein content $(2.1$ and $2.7 \%$, respectively). The difference in casein content is again highlighted in the change of emission spectra shown in Figure 2B. The emission spectral differences among samples I, II, III, and IV illustrate that the tryptophan emission spectra of the acid-precipitated casein were more sensitive to casein content than to $\mathrm{CP}$ content or $\mathrm{CN}: \mathrm{CP}$ in milk.

At $\mathrm{pH}$ 4.6, casein reaches its isoelectric point, aggregating in the milk dispersion system, and the serum phase remains as a transparent liquid. The tryptophancontaining casein aggregates can absorb excitation light $(280 \mathrm{~nm})$ and emit fluorescence at 300 to $400 \mathrm{~nm}$ (Herbert et al., 1999). At the same time, the casein aggregates have surface protuberances that could randomly scatter the excitation light (McMahon et al., 2009). The scattered excitation light may be again absorbed and emit more fluorescence from the casein aggregates. In the serum phase, tryptophan-containing whey proteins, peptides, and free amino acids will also absorb the excitation light and emit fluorescence (Birlouez-Aragon et al., 2002). However, due to the low optical density of the serum phase, less scattering effects could occur. With the scattering of casein aggregates, the tryptophan fluorescence observed in Figure 2 may reflect a change in casein content more than a change in $\mathrm{CP}$ content in a given milk system at a $\mathrm{pH}$ of 4.6.

\section{Calibration Model Development and Optimization}

We constructed PLSR and ENR models using the acid-precipitated casein tryptophan emission spectra as inputs. Because the calibration samples were laboratory-constructed, a validation set was necessary to ensure the model's validity for real milk samples. For PLSR model validation and optimization, the number of latent variables determined the model performance in terms of RMSEV and $\mathrm{R}^{2}$ (Wold et al., 2001).

Model Optimization for PLSR. A typical latent variable selection process is shown in Figure 3A for PLSR prediction of casein content using the Savitzky-Golay smoothing preprocessing technique. The RMSEV $(0.66 \%)$ showed minimal value with 2 latent variables, indicating the lowest prediction error for casein content. The $\mathrm{R}^{2}(0.90)$ had the highest value with 

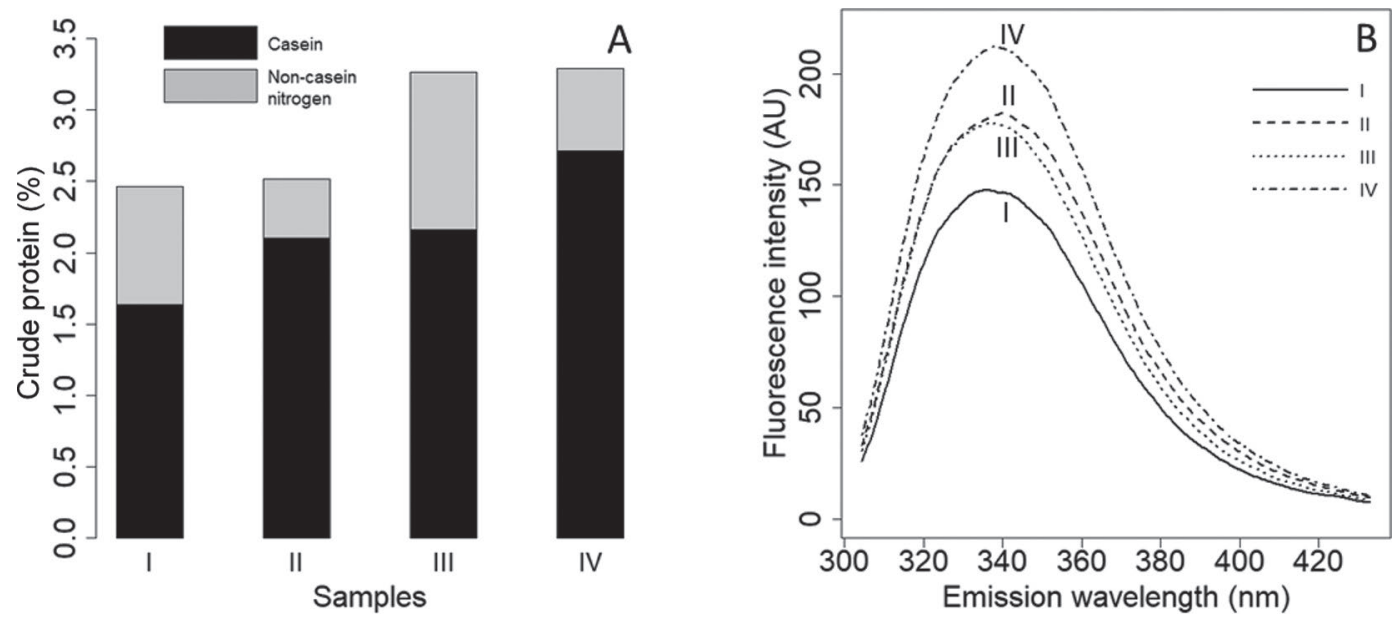

Figure 2. (A) Protein fractions (casein and non-casein nitrogen) of 4 representative calibration samples of low CP (samples I and II) and high CP (samples III and IV). (B) Tryptophan emission spectra of the corresponding samples.

2 latent variables, so we selected the PLSR model with 2 latent variables for the prediction model. However, we observed a high RMSEV from validation, resulting in an RPE of $26.3 \%$. The prediction of the validation set is visualized in Figure 3B. Although we found a good linear trend between reference and predicted casein contents, the high-casein samples showed a proportional overestimation of prediction. Considering the high $\mathrm{R}^{2}$ obtained from the validation, we applied a linear model was applied to correct the overestimation in the validation set. This linear model correction approach is known as calibration transfer. Calibration transfer methods are more commonly seen in the development of near-infrared spectroscopy models to account for instrument signal drifts (Bouveresse et al., 1996; Liu et al., 2016). It is less common to use the calibration transfer method on the same instrument. However, because the calibration samples were laboratory-constructed, they may have yielded different fluorescence intensities because of possible variations in total solids and mineral contents. The validation set was able to capture the high estimation bias and correct it as part of the model development process.

Model Optimization for ENR. To optimize the ENR model, we selected the optimal $\alpha$ using the lowest RMSEV generated from the validation set. In Figure $4 \mathrm{~A}$, a representative elastic net parameter selection process is shown for prediction of casein content using
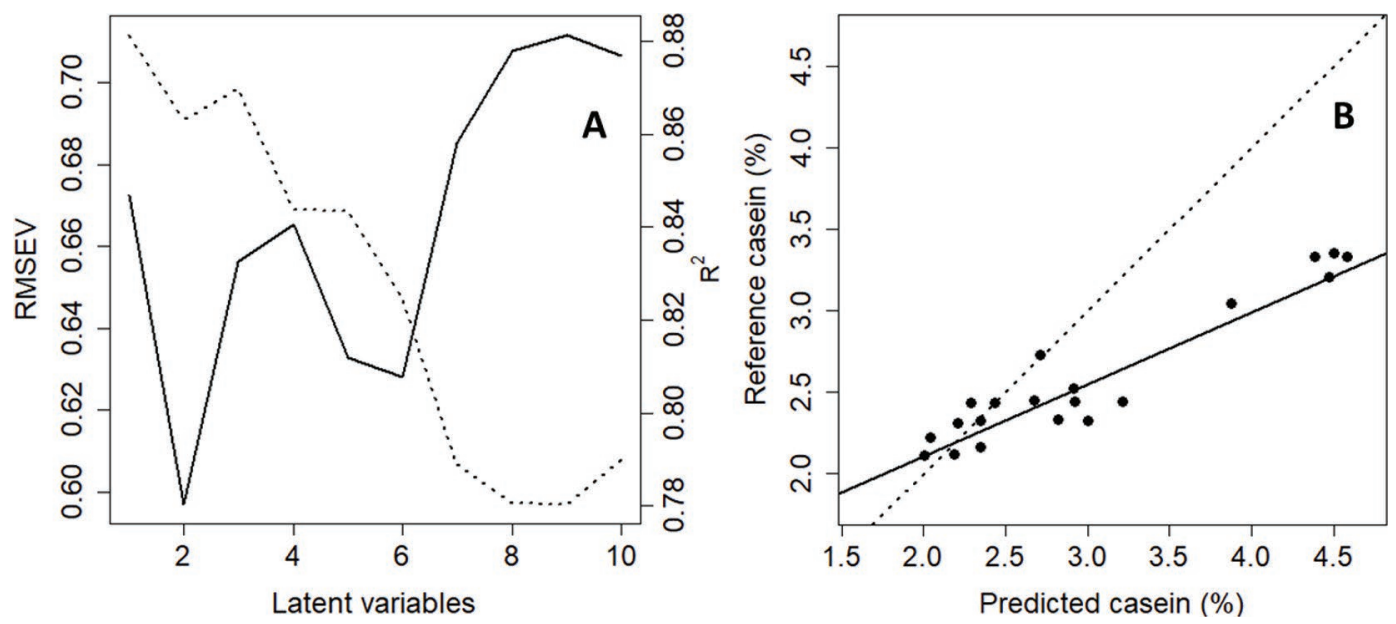

Figure 3. (A) Example of parameter optimization for partial least squares regression (PLSR) for latent variable selections using the validation set. The solid line represents the change in root mean square error of validation (RMSEV), and the dotted line represents the change in coefficient of determination $\left(\mathrm{R}^{2}\right)$. (B) Predicted versus reference casein (\%) of the validation set using PLSR. The solid trend line represents the least square fit of the scatter plot, and the dotted line represents the ideal prediction target $(\mathrm{x}=\mathrm{y})$. 
Table 2. Optimized partial least squares regression (PLSR) and elastic net regression (ENR) calibration model performance of casein and $\mathrm{CN}: \mathrm{CP}$ quantification ${ }^{1}$

\begin{tabular}{|c|c|c|c|c|c|c|c|c|}
\hline Item & Preprocessing & \multicolumn{3}{|c|}{ PLSR } & \multicolumn{4}{|c|}{ ENR } \\
\hline & SG-1st & 0.97 & 0.14 & 2 & 0.97 & 0.17 & 0.30 & 0.33 \\
\hline
\end{tabular}

${ }^{1} \mathrm{RMSEV}=$ root mean square error of validation; Nlv = number of latent variables; SG-S = Savitzky-Golay smoothing; SG-1st $=$ SavitzkyGolay first derivative. $\alpha=$ elastic net parameter; $\lambda=$ regularization parameter.

Savitzky-Golay smoothing as the preprocessing step. The lowest RMSEV observed was $0.64 \%$ when $\alpha$ was 0.9. When $\alpha$ was $0.1, \mathrm{R}^{2}$ reached the highest value of 0.9. When we observed different $\alpha$ values for optimal RMSEV and $\mathrm{R}^{2}$, we chose RMSEV as the evaluating criterion because it judges the true prediction power of the model (Geladi, 2002). Therefore, we chose an $\alpha$ value of 0.9 to optimize the ENR model. As in the PLSR model, we used linear model correction to adjust the biased estimation plotted in Figure $4 \mathrm{~B}$ for ENR predictions.

Optimized Casein Content Prediction Models. Table 2 shows the optimized PLSR and ENR calibration models for casein quantifications, with corresponding optimization factors. For casein content prediction, different preprocessing and regression techniques yielded similar prediction power. The PLSR and ENR models showed similar performance when predicting casein, in agreement with another study that compared PLSR with ENR (Giglio and Brown, 2018). According to Williams and Norris (2001), a $\mathrm{R}^{2}$ value greater than 0.95 indicates reliable model prediction power in food analysis. The low RMSEV for the casein predic- tions also indicated that the model carried potential as a rapid casein quantification method. However, the optimized models needed to be tested externally with unknown samples to estimate their final performance.

\section{External Testing of Casein Quantifications}

The externally tested model performance for casein quantification in UF, skim, and raw milk testing samples is shown in Table 3. For casein prediction, PLSR and ENR yielded similar test results, with RMSEP ranging from 0.12 to $0.13 \%$ and an $\mathrm{R}^{2}$ of 0.91 (Figure $5)$. In further analysis of casein quantification error, the $\mathrm{RPD}$ of the models ranged from 3.2 to 3.4. According to Willams and Norris (2001), an RPD greater than 3 showed very good prediction power for food analysis purposes. The RPE of the casein predictions ranged from 4.9 to $5.1 \%$, showing the relative error in the context of the average casein content of the test set. According to Piñeiro et al. (2008), a linear regression between the reference and predicted casein content showed no significant difference from the slope of 1 and intercept of 0 , indicating that the FFFS-based method
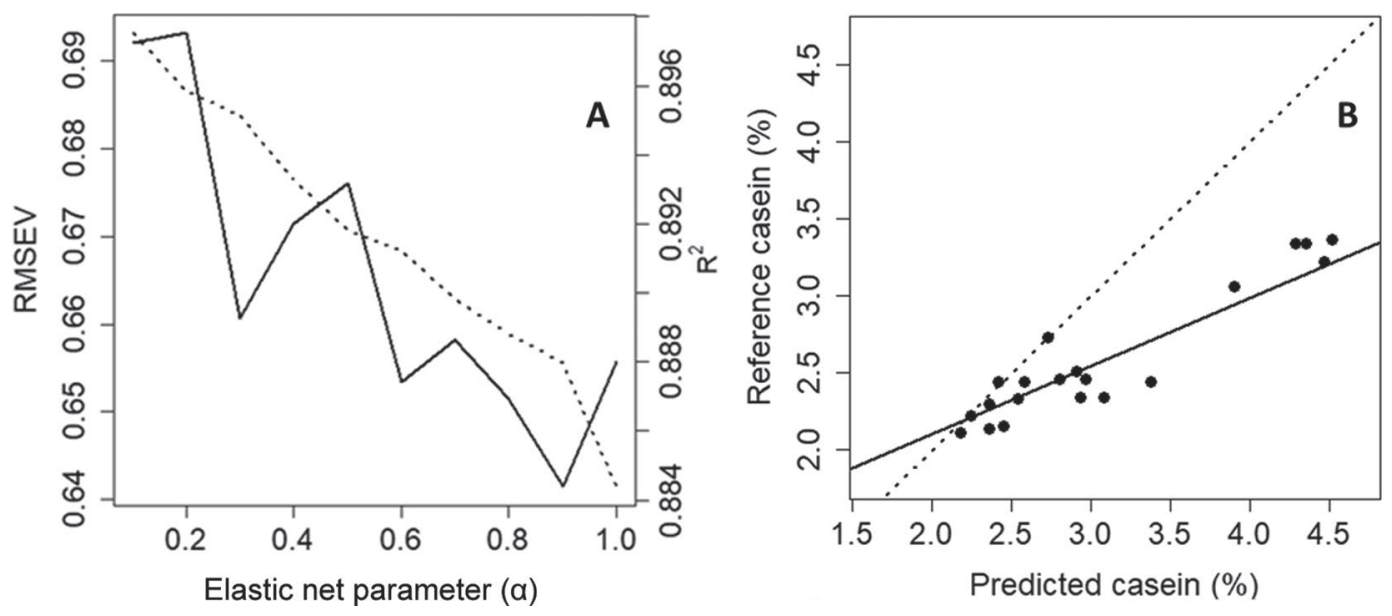

Figure 4. (A) Example of parameter optimization for elastic net regression (ENR) for elastic net parameter selections using the validation set. The solid line represents the change in root mean square error of validation (RMSEV), and the dotted line represents the change in coefficient of determination $\left(\mathrm{R}^{2}\right)$. (B) Predicted versus reference casein (\%) of the validation set using ENR. The solid trend line represents the least square fit of the scatter plot, and the dotted line represents the ideal prediction target $(\mathrm{x}=\mathrm{y})$. 
external validations, the prediction models quantified casein in raw, skim, and UF milk with a RMSEP of 0.12 to $0.13 \%$, an RPD of 3.2 to 3.4 , and an RPE of 4.9 to $5.1 \%$ relative to the reference method. The FFFS-based method provides practical prediction power to serve as a rapid tool for measuring casein content in fluid milk, indicating cheese yield and standardizing cheese milk in dairy farms and processing plants.

\section{ACKNOWLEDGMENTS}

The authors thank MaryAnne Drake from the Southeast Dairy Foods Research Center (Raleigh, $\mathrm{NC}$ ) for the MF retentate and permeate donations. The authors appreciate the technical discussion with Dr. Inès-Birlouez-Aragon from Spectralys Innovation (Romainville, France). This project is Kansas State Research and Extension contribution number 12-278-J. The authors have not stated any conflicts of interest.

\section{REFERENCES}

Andersen, C. M., and G. Mortensen. 2008. Fluorescence spectroscopy: A rapid tool for analyzing dairy products. J. Agric. Food Chem. 56:720-729. https://doi.org/10.1021/jf072025o.

AOAC International. 2016. Official Methods of Analysis. 20th ed. AOAC International, Gaithersburg, MD.

Babu, K. S., and J. K. Amamcharla. 2018. Application of front-face fluorescence spectroscopy as a tool for monitoring changes in milk protein concentrate powders during storage. J. Dairy Sci 101:10844-10859. https://doi.org/10.3168/jds.2018-14885.

Barbano, D. M., and M. E. Dellavalle. 1987. Rapid method for determination of milk casein content by infrared analysis. J. Dairy Sci. 70:1524-1528. https://doi.org/10.3168/jds.S0022-0302(87)80179 -0 .

Barbano, D. M., and J. W. Sherbon. 1984. Cheddar cheese yields in New York. J. Dairy Sci. 67:1873-1883. https://doi.org/10.3168/jds .S0022-0302(84)81517-9.

Baum, A., P. W. Hansen, L. Nørgaard, J. Sørensen, and J. D. Mikkelsen. 2016. Rapid quantification of casein in skim milk using Fourier transform infrared spectroscopy, enzymatic perturbation, and multiway partial least squares regression: Monitoring chymosin at work. J. Dairy Sci. 99:6071-6079. https://doi.org/10.3168/ jds.2016-10947.

Bevilacqua, M., R. Bro, F. Marini, Å. Rinnan, M. A. Rasmussen, and T. Skov. 2017. Recent chemometrics advances for foodomics. TrAC Trend. Anal. Chem. 96:42-51.

Birlouez-Aragon, I., P. Sabat, and N. Gouti. 2002. A new method for discriminating milk heat treatment. Int. Dairy J. 12:59-67. https: //doi.org/10.1016/S0958-6946(01)00131-5.

Bonfatti, V., L. Grigoletto, A. Cecchinato, L. Gallo, and P. Carnier. 2008. Validation of a new reversed-phase high-performance liquid chromatography method for separation and quantification of bovine milk protein genetic variants. J. Chromatogr. A 1195:101106. https://doi.org/10.1016/j.chroma.2008.04.075.

Bouveresse, E., C. Hartmann, D. L. Massart, I. R. Last, and K. A. Prebble. 1996. Standardization of near-infrared spectrometric instruments. Anal. Chem. 68:982-990. https://doi.org/10.1021/ ac9510595.

Brown, C. D., L. Vega-Montoto, and P. D. Wentzell. 2000. Derivative preprocessing and optimal corrections for baseline drift in multivariate calibration. Appl. Spectrosc. 54:1055-1068.

Carter, B., H. Patel, D. M. Barbano, and M. Drake. 2018. The effect of spray drying on the difference in flavor and functional proper- ties of liquid and dried whey proteins, milk proteins, and micellar casein concentrates. J. Dairy Sci. 101:3900-3909. https://doi.org/ $10.3168 /$ jds.2017-13780.

Chen, B., M. J. Lewis, and A. S. Grandison. 2014. Effect of seasonal variation on the composition and properties of raw milk destined for processing in the UK. Food Chem. 158:216-223. https://doi .org/10.1016/j.foodchem.2014.02.118.

Cipolat-Gotet, C., A. Cecchinato, M. De Marchi, and G. Bittante. 2013. Factors affecting variation of different measures of cheese yield and milk nutrient recovery from an individual model cheesemanufacturing process. J. Dairy Sci. 96:7952-7965. https://doi .org/10.3168/jds.2012-6516.

Dimenna, G. P., and H. J. Segall. 1981. High-performance gel-permeation chromatography of bovine skim milk proteins. J. Liq. Chromatogr. 4:639-649. https://doi.org/10.1080/01483918108059961.

Emmons, D. B., and H. W. Modler. 2010. Invited review: A commentary on predictive cheese yield formulas. J. Dairy Sci. 93:55175537. https://doi.org/10.3168/jds.2010-3262.

Filzmoser, P., M. Gschwandtner, and V. Todorov. 2012. Review of sparse methods in regression and classification with application to chemometrics. J. Chemometr. 26:42-51. https://doi.org/10.1002/ cem.1418.

Foss Electric. 1997. Application note No. 102. Calibration for casein in cow milk. MilkoScan FT 120. Foss Electric, Hillerød, Denmark.

Fox, P. F., P. L. McSweeney, and L. H. Paul. 1998. Dairy Chemistry and Biochemistry. Blackie Academic \& Professional, London, UK.

Friedman, J., T. Hastie, and R. Tibshirani. 2010. Regularization paths for generalized linear models via coordinate descent. J. Stat. Softw. 33:1-22. https://doi.org/10.18637/jss.v033.i01.

Geladi, P. 2002. Some recent trends in the calibration literature. Chemom. Intell. Lab. Syst. 60:211-224. https://doi.org/10.1016/S0169 $-7439(01) 00197-6$

Giglio, C., and S. D. Brown. 2018. Using elastic net regression to perform spectrally relevant variable selection. J. Chemometr. 32:e3034. https://doi.org/10.1002/cem.3034.

Herbert, S., A. Riaublanc, B. Bouchet, D. J. Gallant, and E. Dufour. 1999. Fluorescence spectroscopy investigation of acid-or rennetinduced coagulation of milk. J. Dairy Sci. 82:2056-2062. https:// doi.org/10.3168/jds.S0022-0302(99)75446-9.

Hewavitharana, A. K., and B. van Brakel. 1997. Fourier transform infrared spectrometric method for the rapid determination of casein in raw milk. Analyst (Lond.) 122:701-704. https://doi.org/10 $.1039 / \mathrm{a} 700953 \mathrm{~d}$.

Kuhn, M. 2008. Building predictive models in R using the caret package. J. Stat. Softw. 28:1-26

Kulmyrzaev, A. A., D. Levieux, and É. Dufour. 2005. Front-face fluorescence spectroscopy allows the characterization of mild heat treatments applied to milk. Relations with the denaturation of milk proteins. J. Agric. Food Chem. 53:502-507. https://doi.org/ 10.1021/jf049224h.

Kumar, P., N. Sharma, R. Ranjan, S. Kumar, Z. F. Bhat, and D. K. Jeong. 2013. Perspective of membrane technology in dairy industry: A review. Asian-Australas. J. Anim. Sci. 26:1347-1358. https: //doi.org/10.5713/ajas.2013.13082.

Lin, Y., J. A. O'Mahony, A. L. Kelly, and T. P. Guinee. 2017. Seasonal variation in the composition and processing characteristics of herd milk with varying proportions of milk from spring-calving and autumn-calving cows. J. Dairy Res. 84:444-452. https://doi .org/10.1017/S0022029917000516.

Liu, Y., W. Cai, and X. Shao. 2016. Linear model correction: A method for transferring a near-infrared multivariate calibration model without standard samples. Spectrochim. Acta A Mol. Biomol. Spectrosc. 169:197-201. https://doi.org/10.1016/j.saa.2016 .06 .041 .

Lucey, J., and J. Kelly. 1994. Cheese yield. Int. J. Dairy Technol. 47:1-14. https://doi.org/10.1111/j.1471-0307.1994.tb01264.x.

Luginbühl, W. 2002. Evaluation of designed calibration samples for casein calibration in fourier transform infrared analysis of milk. Lebensm. Wiss. Technol. 35:554-558. https://doi.org/10.1006/fstl .2002 .0902 . 
Lundén, A., M. Nilsson, and L. Janson. 1997. Marked effect of $\beta$-lactoglobulin polymorphism on the ratio of casein to total protein in milk. J. Dairy Sci. 80:2996-3005. https://doi.org/10.3168/ jds.S0022-0302(97)76266-0.

Lynch, J. M., D. M. Barbano, and J. R. Fleming. 1998. Indirect and direct determination of the casein content of milk by Kjeldahl nitrogen analysis: Collaborative study. J. AOAC Int. 81:763-774. https://doi.org/10.1093/jaoac/81.4.763.

Ma, Y. B., I. Birlouez-Aragon, and J. K. Amamcharla. 2019. Development and validation of a front-face fluorescence spectroscopy-based method to determine casein in raw milk. Int. Dairy J. 93:81-84. https://doi.org/10.1016/j.idairyj.2019.02.004.

McDermott, A., G. Visentin, M. De Marchi, D. P. Berry, M. A. Fenelon, P. M. O'Connor, O. A. Kenny, and S. McParland. 2016. Prediction of individual milk proteins including free amino acids in bovine milk using mid-infrared spectroscopy and their correlations with milk processing characteristics. J. Dairy Sci. 99:3171-3182. https://doi.org/10.3168/jds.2015-9747.

McMahon, D. J., H. Du, W. R. McManus, and K. M. Larsen. 2009. Microstructural changes in casein supramolecules during acidification of skim milk. J. Dairy Sci. 92:5854-5867. https://doi.org/10 $.3168 /$ jds.2009-2324.

Mevik, B.-H., and R. Wehrens. 2007. The pls Package: principal component and partial least squares regression in R. J. Stat. Softw. 18. https://doi.org/10.18637/jss.v018.i02.

Piñeiro, G., S. Perelman, J. P. Guerschman, and J. M. Paruelo. 2008. How to evaluate models: Observed vs. predicted or predicted vs. observed? Ecol. Modell. 216:316-322. https://doi.org/10.1016/j .ecolmodel.2008.05.006.

R Core Team. 2016. R: A language and environment for statistical computing. R Foundation for Statistical Computing, Vienna, Austria.

Recio, I., and C. Olieman. 1996. Determination of denatured serum proteins in the casein fraction of heat-treated milk by capillary zone electrophoresis. Electrophoresis 17:1228-1233. https://doi .org/10.1002/elps.1150170710.
Rowland, S. J. 1938. 176. The determination of the nitrogen distribution in milk. J. Dairy Res. 9:42-46. https://doi.org/10.1017/ S0022029900002296.

Savitzky, A., and M. J. E. Golay. 1964. Smoothing and differentiation of data by simplified least squares procedures. Anal. Chem. 36:1627-1639. https://doi.org/10.1021/ac60214a047.

Schaar, J., B. Hansson, and H.-E. Pettersson. 1985. Effects of genetic variants of $\kappa$-casein and $\beta$-lactoglobulin on cheesemaking. J. Dairy Res. 52:429-437. https://doi.org/10.1017/S002202990002433X.

Ur-Rehman, S., B. Kopesky, S. Backinoff, T. P. Doelman, and C. White, inventors. 2017. Fractionating milk and UHT sterilization of milk fractions. Fairlife LLC, assignee. U.S. Pat. No. 15/446,032.

van der Ven, C., H. Gruppen, D. B. A. de Bont, and A. G. J. Voragen. 2001. Reversed phase and size exclusion chromatography of milk protein hydrolysates: Relation between elution from reversed phase column and apparent molecular weight distribution. Int. Dairy J. 11:83-92. https://doi.org/10.1016/S0958-6946(01)00032-2.

Walstra, P., and R. Jenness. 1984. Dairy Chemistry and Physics. John Wiley \& Sons, Hoboken, NJ.

Williams, P. C., and K. Norris. 2001. Near-Infrared Technology in the Agricultural and Food Industry. American Association of Cereal Chemists, Inc., St. Paul, MN

Wold, S., M. Sjöström, and L. Eriksson. 2001. PLS-regression: A basic tool of chemometrics. Chemom. Intell. Lab. Syst. 58:109-130. https://doi.org/10.1016/S0169-7439(01)00155-1.

Zhang, H., and L. E. Metzger. 2011. Noncasein nitrogen analysis of ultrafiltration and microfiltration retentate. J. Dairy Sci. 94:21182125. https://doi.org/10.3168/jds.2010-3690.

\section{ORCIDS}

Jayendra. K. Amamcharla ( https://orcid.org/0000-0001-6620-0089 